ESAIM: PROCEEDINGS, May 2009, Vol. 27, p. 107-121

C. Besse, O. Goubet, T. Goudon \& S. Nicaise, Editors

\title{
A POSTERIORI ERROR ANALYSIS FOR POISSON'S EQUATION APPROXIMATED BY XFEM*
}

\author{
Patrick Hild ${ }^{1}$, VAnessa Lleras ${ }^{2}$ And Yves Renard ${ }^{3}$
}

\begin{abstract}
This paper presents and studies a residual a posteriori error estimator for Laplace's equation in two space dimensions approximated by the eXtended Finite Element Method (XFEM). The XFEM allows to perform finite element computations on multi-cracked domains by using meshes of the non-cracked domain. The main idea consists of adding supplementary basis functions of Heaviside type and singular functions in order to take into account the crack geometry and the singularity at the crack tip respectively.
\end{abstract}

Résumé. Dans ce travail on propose et on étudie un estimateur d'erreur par résidu pour l'équation de Laplace en deux dimensions d'espace discrétisée par la méthode d'éléments finis étendue (XFEM). La XFEM permet de réaliser des simulations par éléments finis sur des domaines multi-fissurés en utilisant des maillages du domaine non fissuré. L'idée principale de la méthode consiste à ajouter des fonctions de base supplémentaires de type Heaviside et des fonctions singulières afin de prendre en compte la géométrie de la fissure et la singularité en pointe de fissure.

\section{INTRODUCTION AND NOTATION}

The eXtended Finite Element Method (XFEM) was introduced in $[20,21]$ (in the linear elasticity context) in order to avoid remeshing in domains with evolutionary cracks. The idea of the method is to enrich the classical finite element basis with both non-smooth functions representing the singularities at the reentrant corners (as in the singular enrichment method introduced in [24]) and also with step functions (of Heaviside type) along the crack since the finite element mesh does not coincide with the cracked domain. After numerous numerical works developed in various contexts of mechanics, the first convergence results with a priori error estimates (in linear elasticity) were recently obtained in [6,7]: in the convergence analysis, a difficulty consists in evaluating the local error in the elements cut by the crack by using appropriate extension operators and specifical estimates. In the latter references, the authors obtain an error estimate of order $h$ ( $h$ denotes the discretization parameter) under $H^{2+\varepsilon}$ regularity of the regular part of the solution keeping in mind that the solution is only $H^{3 / 2-\varepsilon}$ regular.

In the present work we propose and analyze an error estimator of residual type (see [2] for the early ideas and analyzes and e.g., [25] and the references therein for a more complete overview) for the XFEM in the simple framework of Laplace equation. Since the meshes do not coincide with the domain we need to introduce and

\footnotetext{
* This work is supported by "l'Agence Nationale de la Recherche", project ANR-05-JCJC-0182-01.

1 Laboratoire de Mathématiques de Besançon, UMR CNRS 6623, Université de Franche-Comté, 16 route de Gray, 25030 Besançon, France; e-mail: patrick.hild@univ-fcomte.fr

2 Laboratoire de Mathématiques de Besançon, UMR CNRS 6623, Université de Franche-Comté, 16 route de Gray, 25030 Besançon, France; e-mail: vanessa.lleras@univ-fcomte.fr

3 Institut Camille Jordan, UMR CNRS 5208, INSA de Lyon, 20 rue Albert Einstein, 69621 Villeurbanne, France; e-mail: yves.renard@insa-lyon.fr

(C) EDP Sciences, SMAI 2009
} 
to study a new quasi-interpolation operator of averaging type (see e.g., $[3,8,10,18,22,23]$ for various averaging type operators). The use of the new operator allows us to perform a first a posteriori error analysis.

We introduce some useful notation and several functional spaces. As usual, we denote by $\left(L^{2}(.)\right)^{d}$ and by $\left(H^{s}(.)\right)^{d}, s \geq 0, d=1,2$ the Lebesgue and Sobolev spaces in one and two space dimensions (see [1]). The usual norm of $\left(H^{s}(D)\right)^{d}$ is denoted by $\|\cdot\|_{s, D}$ and we keep the same notation when $d=1$ or $d=2$. For shortness the $\left(L^{2}(D)\right)^{d}$-norm will be denoted by $\|\cdot\|_{D}$ when $d=1$ or $d=2$. In the sequel the symbol $|\cdot|$ will denote either the Euclidean norm in $\mathbb{R}^{2}$, or the length of a line segment, or the area of a plane domain. Finally the notation $a \lesssim b$ means here and below that there exists a positive constant $C$ independent of $a$ and $b$ (and of the meshsize of the triangulation) such that $a \leq C b$. The notation $a \sim b$ means that $a \lesssim b$ and $b \lesssim a$ hold simultaneously.

\section{The Poisson problem on a CRACKed domain}

Let $\Omega$ be a open subset of $\mathbb{R}^{2}$ having a crack, with a polygonal boundary $\partial \Omega$ where $\Gamma_{C} \subset \partial \Omega$ denotes the crack (the crack $\Gamma_{C}$ consists of two distinct straight line segments having the same location). We fix a "partition" of $\partial \Omega$ into three open disjoint subsets $\Gamma_{D}, \Gamma_{N}$ and $\Gamma_{C}$ where we will consider homogeneous Dirichlet (on $\left.\Gamma_{D}\right)$ and homogeneous Neumann (on $\Gamma_{N} \cup \Gamma_{C}$ ) boundary conditions respectively. We choose homogeneous conditions to simplify the notation and the extension to nonhomogeneous conditions can be made without additional difficulties. We assume that $\partial \Omega=\bar{\Gamma}_{D} \cup \bar{\Gamma}_{N} \cup \bar{\Gamma}_{C}$. We further suppose that the measures of $\Gamma_{D}$ and $\Gamma_{C}$ are positive (see Figure 1).

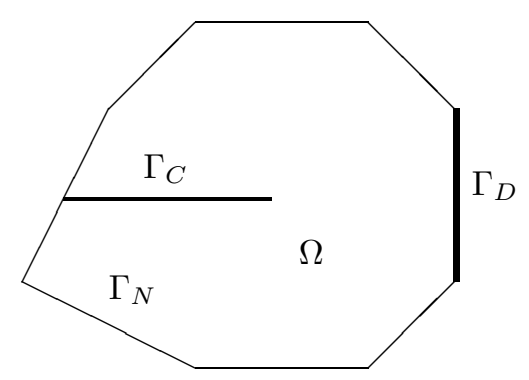

FiguRE 1. The geometry of the cracked domain $\Omega$

In this paper we consider the Poisson problem: for $f \in L^{2}(\Omega)$ let $u \in H^{1}(\Omega)$ be the variational solution of

$$
\left\{\begin{array}{lll}
-\Delta u & =f & \text { in } \Omega \\
u & =0 & \text { on } \Gamma_{D} \\
\partial_{n} u & =0 & \text { on } \Gamma_{N} \cup \Gamma_{C}
\end{array}\right.
$$

where $\partial_{n} u=\nabla u \cdot n=\partial u / \partial n$ means the outward normal derivative of $u$ along the boundary and $n$ stands for the unit outward normal vector of $\partial \Omega$. We choose Laplace operator instead of the elasticity operator to avoid supplementary notation keeping in mind that the XFEM was intensively studied in the elasticity context. Set

$$
V=\left\{u \in H^{1}(\Omega): u=0 \text { on } \Gamma_{D}\right\}
$$

Then the variational solution of (1) is the unique solution $u$ of

$$
u \in V, \quad \int_{\Omega} \nabla u \cdot \nabla v d x=\int_{\Omega} f v d x, \quad \forall v \in V .
$$


The solution $u$ to the Poisson problem can be written as a sum of a singular part $u_{s}=K r^{1 / 2} \sin (\theta / 2)$ and a regular part $u-u_{s}$ (see, e.g, $[15,16]$ ) where $K \in \mathbb{R}$ is the stress intensity factor and the notation $(r, \theta)$ denotes the polar coordinates with respect to the crack tip (the value $\theta=\pi$ corresponds to the crack so that $u_{s}$ is discontinuous across the crack). It can be checked that for any positive $\varepsilon, u_{s}$ lies in $H^{\frac{3}{2}-\varepsilon}(\Omega)$ (see, e.g. [15,16]).

\section{Discretizhtion of the Poisson problem with the XFEM}

We approximate problem (1) by the so called XFEM (eXtended Finite Element Method) introduced in [21]. Namely we consider a regular family of triangulations $T_{h}, h>0$ of the noncracked domain made of closed triangles $T$ such that $\bar{\Omega}=\cup_{T \in T_{h}} T$ (see $[5,9,11]$ ). For $T \in T_{h}$ we recall that $h_{T}$ is the diameter of $T$ and $h=\max _{T \in T_{h}} h_{T}$. The regularity of the mesh implies in particular that for any edge $E$ of $T$ one has $h_{E}=|E| \sim h_{T}$. Since the triangles in $T_{h}$ do not coincide with the geometry of the body on the crack we define the family of generalized elements $G_{h}, h>0$ containing the following elements (see Figure 2):

- the triangles in $T \in T_{h}$ whose interior does not intersect $\Gamma_{C}$,

- the (non closed) triangles and quadrangles obtained when the crack cuts (in two parts) a triangle in $T_{h}$,

- the (non closed) cracked triangle containing the crack tip.
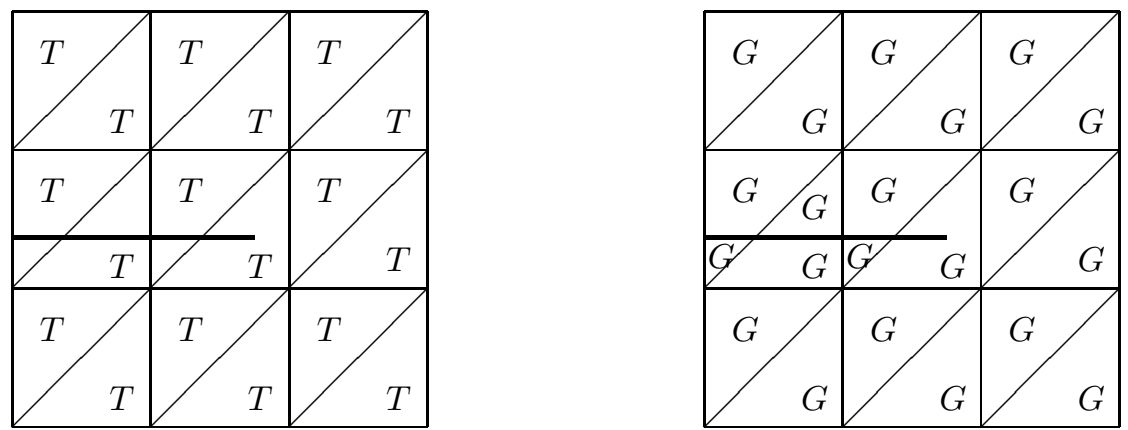

Figure 2. Standard elements $T \in T_{h}$ and generalized elements $G \in G_{h}$

This implies that $\bar{\Omega}=\cup_{G \in G_{h}} \bar{G}$ and $\cup_{G \in G_{h}} G^{\circ} \subset \Omega$ where $G^{\circ}$ denotes the largest open set contained in $G$. We next give an important definition:

Definition 3.1. Let $\mathcal{N}_{h}$ be the set of nodes of the triangulation $T_{h}$. We say that a node $x \in \mathcal{N}_{h}$ is enriched if the patch surrounding $x: \omega_{x}=\cup_{T: x \in T_{h}} T$ is cut in (at least) two subsets by the crack (see Figure 3) and we denote by $\mathcal{N}_{h}^{H} \subset \mathcal{N}_{h}$ the set of enriched nodes. We say that a triangle is enriched (resp. partially enriched) if its three nodes (resp. one or two nodes) are enriched.

We denote by $h_{x}$ the diameter of the patch $\omega_{x}$. If $T \in T_{h}$ we denote by $\omega_{T}$ the union of all elements in $T_{h}$ having a nonempty intersection with $T$. Similarly for a edge $E$ of a triangle in $T_{h}$ we denote by $\omega_{E}$ the union of all elements in $T_{h}$ having a nonempty intersection with $\bar{E}$. Set $\mathcal{N}_{h}^{D}=\mathcal{N}_{h} \cap \bar{\Gamma}_{D}$ (note that the extreme nodes of $\bar{\Gamma}_{D}$ belong to $\mathcal{N}_{h}^{D}$ ).

Let $E_{h}$ denote the set of edges of the elements in $G_{h}$ (the edges are supposed to be relatively open). Set $E_{h}^{i n t}=\left\{E \in E_{h}: E \subset \Omega\right\}$ the set of interior edges of $G_{h}, E_{h}^{\text {ext }}=E_{h} \backslash E_{h}^{\text {int }}$. We denote by $E_{h}^{N}=\left\{E \in E_{h}\right.$ : $\left.E \subset \Gamma_{N}\right\}, E_{h}^{C}=\left\{E \in E_{h}: E \subset \Gamma_{C}\right\}$ the set of exterior edges included into the part of the boundary where we impose Neumann conditions. For a generalized element $G \in G_{h}$ (resp. standard element $T \in T_{h}$ ), we will denote by $E_{G}$ the set of edges of $G$ (resp., by $E_{T}$ the set of edges of $T$ ) and according to the above notation, we set $E_{G}^{i n t}=E_{G} \cap E_{h}^{i n t}, E_{G}^{N}=E_{G} \cap E_{h}^{N}, E_{G}^{C}=E_{G} \cap E_{h}^{C}$. For an edge $E$ of a generalized element $G$ we introduce $n_{G, E}=\left(n_{x}, n_{y}\right)$ the unit outward normal vector to $G$ along $E$. Furthermore for each edge $E \in E_{h}$ we fix one 


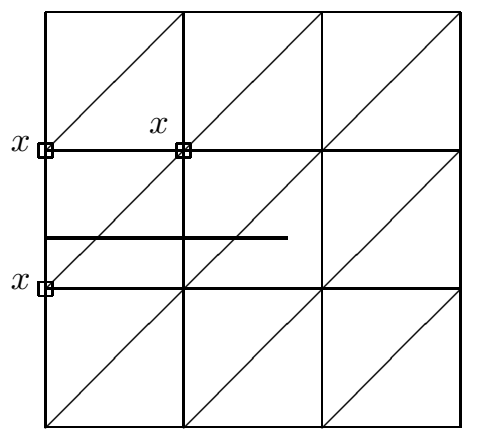

FiguRE 3. Enriched nodes $x \in \mathcal{N}_{h}^{H}$

of the two normal vectors and denote it by $n_{E}$. The jump of some function $v$ across an edge $E \in E_{h}$ at a point $y \in E$ is defined as

$$
\llbracket v(y) \rrbracket_{E}=\left\{\begin{array}{cc}
\lim _{\alpha \rightarrow 0^{+}} v\left(y+\alpha n_{E}\right)-v\left(y-\alpha n_{E}\right) & \forall E \in E_{h}^{i n t}, \\
v(y) & \forall E \in E_{h}^{e x t} .
\end{array}\right.
$$

The main idea of the extended finite element method is to enrich the classical finite element space by both:

- a singular function at the crack tip in order to take into account the corner singularity (note that in the vector valued case, as elasticity, several singular functions have to be added),

- discontinuous functions located near the crack in order to take into account the discontinuity of the solution across the crack.

The latter discontinuous functions are constructed using the Heaviside function $H$ which is equal to 1 on one side (of the straight extension, see Figure 4) of the crack and to -1 on the other side.

We denote by $\lambda_{x}$ the classical finite element $P_{1}$ basis functions at node $x \in \mathcal{N}_{h}$ satisfying $\lambda_{x}\left(x^{\prime}\right)=\delta_{x, x^{\prime}}$, for any $x^{\prime} \in \mathcal{N}_{h}$. The extended finite element space is defined as follows:

$$
\begin{aligned}
V_{h}=\left\{v_{h} \in C(\Omega): v_{h}\right. & =\sum_{x \in \mathcal{N}_{h}} a_{x} \lambda_{x}+\sum_{x \in \mathcal{N}_{h}^{H}} b_{x} H \lambda_{x}+c \chi r^{1 / 2} \sin (\theta / 2) \\
& \left.=v_{h, r}+\chi v_{h, s}, \quad a_{x}, b_{x}, c \in \mathbb{R}\right\} \subset V,
\end{aligned}
$$

where $\chi \in C^{2}(\bar{\Omega})$ is a cutoff function defined as follows. Let be given $0<r_{0}<r_{1}$ and denoting by $r$ the distance to the crack tip, we have:

$$
\begin{cases}\chi(r)=1 & \text { if } r \leq r_{0} \\ 0<\chi(r)<1 & \text { if } r_{0}<r<r_{1} \\ \chi(r)=0 & \text { if } r \geq r_{1}\end{cases}
$$

The cutoff function $\chi$ was introduced in $[6,7]$ in order to improve the performances (in terms of convergence) of the original method introduced in [21].

The finite element problem issued from (2) consists of finding $u_{h}$ such that

$$
u_{h} \in V_{h}, \quad \int_{\Omega} \nabla u_{h} \cdot \nabla v_{h} d x=\int_{\Omega} f v_{h} d x, \quad \forall v_{h} \in V_{h} .
$$

Using the Lax-Milgram Lemma, we deduce that problem (4) admits a unique solution.

Remark 3.2. A quasi-optimal a priori error estimate is obtained in [6, 7] for the XFEM involving the cutoff function $\chi$. Under $H^{2+\varepsilon}(\Omega)$ regularity for $u-u_{s}$, the authors prove that $\left\|u-u_{h}\right\|_{1, \Omega} \lesssim h\left\|u-u_{s}\right\|_{2+\varepsilon, \Omega}$. Note 
that when using a classical finite element method we obtain a convergence rate of only $h^{1 / 2-\varepsilon}$ since $u$ lies in $H^{3 / 2-\varepsilon}(\Omega)$.

\section{The QUASI-INTERPOLATION OPERATOR}

\subsection{Definition}

In order to perform a residual a posteriori error analysis one currently uses a quasi-interpolation operator $\pi_{h}: V \rightarrow V_{h}$ of averaging type (see e.g., $[3,8,10,18,22,23]$ for various operators). At a node $x$, the value of the quasi-interpolation is often an "average" of the function on the patch $\omega_{x}$ surrounding $x$. To simplify the forthcoming discussion we suppose (as in Figure 1) that the end points of the crack belonging to $\partial \bar{\Omega}$ are not submitted to Dirichlet conditions.

So the first idea would be to use such an operator on the regular mesh $T_{h}$. In such an analysis there are terms such as $\left\|u-u_{h}\right\|_{1, \omega_{T}}$ (where $T \in T_{h}$ ) which appear and unfortunately $u$ does not lie in $H^{1}(\bar{\Omega})$ (hence $u-u_{h}$ does in general not lie in $\left.H^{1}\left(\omega_{T}\right)\right)$ due to the discontinuity across the crack.

A second idea would be to define $\pi_{h} v$ (with $v \in H^{1}(\Omega)$ ) separately on each side of the crack. If we divide $\Omega$ into $\Omega_{1}$ and $\Omega_{2}$ using the crack and a straight extension of the crack (see Figure 4 ) one could first try to define $\pi_{h} v$ on $\Omega_{1}$ (resp. $\Omega_{2}$ ) by using the only values of $v$ on $\Omega_{1}$ (resp. $\Omega_{2}$ ). This consists of defining $\pi_{h} v$ on each generalized element $G \in G_{h}$. It is easy to see that this approach leads to technical difficulties since the elements in $G_{h}$ are sometimes quadrangles and so on.

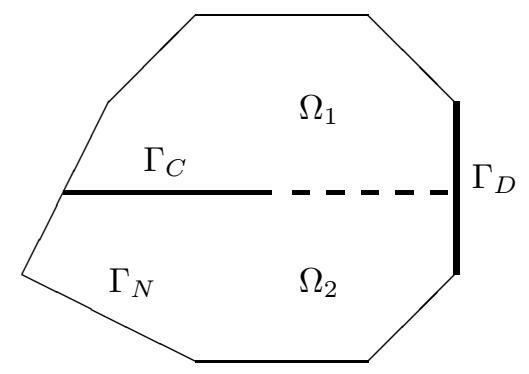

FiguRE 4. Domain decomposition using a straight extension of the crack

So we choose an approach which consists to determine $\pi_{h} v$ separately on each side of the crack by defining $\pi_{h} v_{\left.\right|_{G}}, G \in G_{h}$ and by using the values of $v$ on both sides of the crack. This leads us to use extension operators. As already mentioned, let us divide $\Omega$ into $\Omega_{1}$ and $\Omega_{2}$ using the crack and a straight extension of the crack (see Figure 4). Let $v \in H^{1}(\Omega)$ with $v_{1}=v_{\Omega_{1}} \in H^{1}\left(\Omega_{1}\right)$ and $v_{2}=v_{\left.\right|_{\Omega_{2}}} \in H^{1}\left(\Omega_{2}\right)$. We define an extension of $v_{1}$ defined on $\Omega$ (see $[1,12]$ ) denoted $\tilde{v}_{1}$ such that

$$
\left\|\tilde{v}_{1}\right\|_{1, \Omega} \lesssim\left\|v_{1}\right\|_{1, \Omega_{1}} \leq\|v\|_{1, \Omega}
$$

and an extension $\tilde{v}_{2}$ (defined on $\Omega$ ) of $v_{2}$ such that

$$
\left\|\tilde{v}_{2}\right\|_{1, \Omega} \lesssim\left\|v_{2}\right\|_{1, \Omega_{2}} \leq\|v\|_{1, \Omega}
$$

where the constant $C$ depends neither on $v_{1}$ nor on $v_{2}$.

For any $v \in H^{1}(\Omega)$, we define $\pi_{h} v$ as the unique element in $V_{h}$ such that

$$
\pi_{h} v=\sum_{x \in \mathcal{N}_{h}} \alpha_{x}(v) \lambda_{x}+\sum_{x \in \mathcal{N}_{h}^{H}} \beta_{x}(v) H \lambda_{x} .
$$


Remark 4.1. Note that $\pi_{h}(V) \subset V_{h}$ with $\pi_{h}(V) \neq V_{h}$ since the singular function at the crack tip does not belong to the range of $\pi_{h}$.

- Step 1. Definition of $\pi_{h} v$ at the nodes $\mathcal{N}_{h}$ of the triangulation $T_{h}$.

(i: not enriched nodes) If $x \in \mathcal{N}_{h} \backslash \mathcal{N}_{h}^{D}$ is such that $\omega_{x}$ is not cut (i.e., not divided into more than one part) by the crack then

$$
\pi_{h} v(x)=\frac{1}{\left|\omega_{x}\right|} \int_{\omega_{x}} v(y) d y .
$$

From Cauchy Schwarz inequality, we get

$$
\left|\pi_{h} v(x)\right| \lesssim\left|\omega_{x}\right|^{-1 / 2}\|v\|_{\omega_{x}} \sim h_{x}^{-1}\|v\|_{\omega_{x}} \leq h_{x}^{-1}\|v\|_{\omega_{x}}+\|\nabla v\|_{\omega_{x}}
$$

Note that the three nodes of the triangle containing the crack tip are concerned with the latter case (see Figure $3)$.

(ii: enriched nodes) If $x \in \mathcal{N}_{h} \backslash \mathcal{N}_{h}^{D}, x \in \bar{\Omega}_{\ell}, \ell=1,2$, is such that $\omega_{x}$ is cut by the crack then we set

$$
\pi_{h} v(x)=\frac{1}{\left|\omega_{x}\right|} \int_{\omega_{x}} \tilde{v}_{\ell}(y) d y .
$$

Note that if $x$ lies on the crack and (ii) is satisfied then there are two values of $\pi_{h} v(x)$ : one corresponding to $\pi_{h} v(x)$ on $\bar{\Omega}_{1}$ and the other one to $\pi_{h} v(x)$ on $\bar{\Omega}_{2}$. So we deduce

$$
\left|\pi_{h} v(x)\right| \lesssim\left|\omega_{x}\right|^{-1 / 2}\left\|\tilde{v}_{\ell}\right\|_{\omega_{x}} \sim h_{x}^{-1}\left\|\tilde{v}_{\ell}\right\|_{\omega_{x}} \leq h_{x}^{-1}\left\|\tilde{v}_{\ell}\right\|_{\omega_{x}}+\left\|\nabla \tilde{v}_{\ell}\right\|_{\omega_{x}}
$$

(iii) If $x \in \mathcal{N}_{h}^{D}$, denote $\Gamma_{x}=\omega_{x} \cap \Gamma_{D}$ (recall that $\bar{\Gamma}_{C} \cap \bar{\Gamma}_{D}=\emptyset$ to simplify the discussion) and set:

$$
\pi_{h} v(x)=\frac{1}{\left|\Gamma_{x}\right|} \int_{\Gamma_{x}} v(y) d \Gamma .
$$

By using a scaled trace inequality (see, e.g., $[15,17])$ :

$$
\|v\|_{E} \lesssim h_{E}^{-1 / 2}\|v\|_{T}+h_{E}^{1 / 2}\|\nabla v\|_{T}, \quad \forall v \in H^{1}(T), \forall T \in T_{h}, \forall E \in E_{T}
$$

we get

$$
\begin{aligned}
\left|\pi_{h} v(x)\right| \lesssim\left|\Gamma_{x}\right|^{-1 / 2}\|v\|_{\Gamma_{x}} \sim h_{x}^{-1 / 2}\|v\|_{\Gamma_{x}} & \lesssim h_{x}^{-1 / 2}\left(h_{x}^{-1 / 2}\|v\|_{\omega_{x}}+h_{x}^{1 / 2}\|\nabla v\|_{\omega_{x}}\right) \\
& \lesssim h_{x}^{-1}\|v\|_{\omega_{x}}+\|\nabla v\|_{\omega_{x}}
\end{aligned}
$$

- Step 2. Definition of $\pi_{h} v$ on $\Omega$.

With the previous nodal expressions we define by linear interpolation the function $\pi_{h} v$ on any triangle excepted those cut by the crack. Note that a triangle can be totally enriched (i.e., its three nodes are enriched) and not cut by the crack. If the triangle is cut by the crack then it is either enriched (three nodes enriched) or partially enriched (one or two enriched nodes). The definition on the triangles cut by the crack is given hereafter.

Consider first a totally enriched triangle $T$ with e.g., $x_{1} \in \Omega_{1}$ and $x_{2}, x_{3} \in \Omega_{2}$. In order to determine $\left(\pi_{h} v\right)_{\left.\right|_{\Omega_{1} \cap T}}$, we write:

$$
\pi_{h} v(x)=\frac{1}{\left|\omega_{x}\right|} \int_{\omega_{x}} \tilde{v}_{1}(y) d y .
$$

for $x=x_{1}, x_{2}, x_{3}$. Then $\pi_{h} v$ is defined by linear interpolation on $T$ and then we restrict $\pi_{h} v$ to $T \cap \Omega_{1}$. 
Similarly we define $\left(\pi_{h} v\right)_{\left.\right|_{\Omega_{2} \cap T}}$ by linear interpolation on $T$ by using:

$$
\pi_{h} v(x)=\frac{1}{\left|\omega_{x}\right|} \int_{\omega_{x}} \tilde{v}_{2}(y) d y .
$$

for $x=x_{1}, x_{2}, x_{3}$ and then we restrict the function to $T \cap \Omega_{2}$.

A similar construction is achieved for the partially enriched triangles: if a node $x$ is not enriched then we compute the value of $\pi_{h} v(x)$ at this node and if it is enriched then we compute both quantities (9) and (10) corresponding to $\tilde{v}_{1}$ and $\tilde{v}_{2}$ at this node.

Remark 4.2. From the previous construction of $\pi_{h} v$ and expression (7) we see that $\alpha_{x}(v)=\pi_{h} v(x)$ if $x \in$ $\mathcal{N}_{h} \backslash \mathcal{N}_{h}^{H}$. If $x \in \mathcal{N}_{h}^{H}, x \in \bar{\Omega}_{k}$ and denoting $\ell=3-k$, we have

$$
\alpha_{x}(v)+\beta_{x}(v) H(x)=\frac{1}{\left|\omega_{x}\right|} \int_{\omega_{x}} \tilde{v}_{k}(y) d y, \quad \text { and } \quad \alpha_{x}(v)-\beta_{x}(v) H(x)=\frac{1}{\left|\omega_{x}\right|} \int_{\omega_{x}} \tilde{v}_{\ell}(y) d y .
$$

Hence

$$
\alpha_{x}(v)=\frac{1}{2\left|\omega_{x}\right|} \int_{\omega_{x}} \tilde{v}_{k}(y)+\tilde{v}_{\ell}(y) d y, \quad \text { and } \quad \beta_{x}(v)=\frac{H(x)}{2\left|\omega_{x}\right|} \int_{\omega_{x}} \tilde{v}_{k}(y)-\tilde{v}_{\ell}(y) d y
$$

\subsection{Stability}

Next we consider the stability properties on the generalized elements:

Lemma 4.3. For all $v \in H^{1}(\Omega)$ and all $T \in T_{h}$ one has:

(i) if none of the nodes of $T$ is enriched (so the crack does not cut $T$ ) then:

$$
\left\|\pi_{h} v\right\|_{T} \lesssim\|v\|_{\omega_{T}}+h_{T}\|\nabla v\|_{\omega_{T}}
$$

(ii) if the three nodes of $T$ are enriched, then for $\ell=1$ and $\ell=2$, we have:

$$
\left\|\pi_{h} v\right\|_{T \cap \Omega_{\ell}} \lesssim\left\|\tilde{v}_{\ell}\right\|_{\omega_{T}}+h_{T}\left\|\nabla \tilde{v}_{\ell}\right\|_{\omega_{T}}
$$

(iii) if one or two nodes of $T$ are enriched and if $\omega_{T}$ is cut by the crack (so $T \subset \bar{\Omega}_{\ell}$ for $\ell=1$ or $\left.\ell=2\right)$ we have:

$$
\left\|\pi_{h} v\right\|_{T} \lesssim\left\|\tilde{v}_{\ell}\right\|_{\omega_{T}}+h_{T}\left\|\nabla \tilde{v}_{\ell}\right\|_{\omega_{T}}
$$

(iv) if one or two nodes of $T$ are enriched and if $\omega_{T}$ contains the crack tip then for $\ell=1$ or $\ell=2$, we have:

$$
\left\|\pi_{h} v\right\|_{T \cap \Omega_{\ell}} \lesssim\left\|\tilde{v}_{\ell}\right\|_{\omega_{T}}+\|v\|_{\omega_{T}}+h_{T}\left\|\nabla \tilde{v}_{\ell}\right\|_{\omega_{T}}+h_{T}\|\nabla v\|_{\omega_{T}}
$$

Remark 4.4. Due to the mesh regularity there is a mesh independent bounded number of triangles satisfying (iv): more precisely this set is contained in $\omega_{T^{*}}$ where $T^{*}$ is the triangle containing the crack tip. Some of the triangles in this set are cut by the crack and others no.

Proof: (i). If none of the nodes is enriched then for any of the three nodes of $T$, we have:

$$
\left|\pi_{h} v(x)\right| \lesssim h_{x}^{-1}\|v\|_{\omega_{x}}+\|\nabla v\|_{\omega_{x}}
$$

Writing $\pi_{h} v=\sum_{x \in T} \pi_{h} v(x) \lambda_{x}$ on $T$ and using $\left\|\lambda_{x}\right\|_{T} \sim h_{T} \sim h_{x}$ implies the result.

(ii). Set e.g., $\ell=1$. Noting that for any of the three node of $T$, we have

$$
\left|\pi_{h} v(x)\right| \lesssim h_{x}^{-1}\left\|\tilde{v}_{1}\right\|_{\omega_{x}}+\left\|\nabla \tilde{v}_{1}\right\|_{\omega_{x}},
$$

and using the same estimates as in (i) yields the result. The same result holds when $\ell=2$.

(iii) and (iv). These estimates are obtained as the previous ones. 
Now we consider the stability properties of $\pi_{h}$ on the edges of the generalized elements not located on the crack.

Lemma 4.5. For all $v \in H^{1}(\Omega)$ and all edge $E$ of a triangle $T \in T_{h}$ one has:

(i) if both end points of $E$ are not enriched:

$$
\left\|\pi_{h} v\right\|_{E} \lesssim h_{E}^{-1 / 2}\|v\|_{\omega_{E}}+h_{E}^{1 / 2}\|\nabla v\|_{\omega_{E}}
$$

(ii) if both end points of $E$ are enriched then for $\ell=1$ or $\ell=2$, we have:

$$
\left\|\pi_{h} v\right\|_{E \cap \bar{\Omega}_{\ell}} \lesssim h_{E}^{-1 / 2}\left\|\tilde{v}_{\ell}\right\|_{\omega_{E}}+h_{E}^{1 / 2}\left\|\nabla \tilde{v}_{\ell}\right\|_{\omega_{E}}
$$

(iii) if only one end point of $E$ is enriched and if $\omega_{E}$ is cut by the crack (so $E \subset \bar{\Omega}_{\ell}$ for $\ell=1$ or $\ell=2$ ), we have:

$$
\left\|\pi_{h} v\right\|_{E} \lesssim h_{E}^{-1 / 2}\left\|\tilde{v}_{\ell}\right\|_{\omega_{E}}+h_{E}^{1 / 2}\left\|\nabla \tilde{v}_{\ell}\right\|_{\omega_{E}}
$$

(iv) if only one end point of $E$ is enriched and if $\omega_{E}$ contains the crack tip then for $\ell=1$ or $\ell=2$, we have:

$$
\left\|\pi_{h} v\right\|_{E \cap \bar{\Omega}_{\ell}} \lesssim h_{E}^{-1 / 2}\left\|\tilde{v}_{\ell}\right\|_{\omega_{E}}+h_{E}^{-1 / 2}\|v\|_{\omega_{E}}+h_{E}^{1 / 2}\left\|\nabla \tilde{v}_{\ell}\right\|_{\omega_{E}}+h_{E}^{1 / 2}\|\nabla v\|_{\omega_{E}}
$$

Remark 4.6. There is a meshsize independent bounded number of edges satisfying (iv). More precisely all these edges have an end point belonging to the triangle containing the crack tip.

Proof: (i). For both end points of $E$, we have:

$$
\left|\pi_{h} v(x)\right| \lesssim h_{x}^{-1}\|v\|_{\omega_{x}}+\|\nabla v\|_{\omega_{x}}
$$

Writing $\pi_{h} v=\sum_{x \in E} \pi_{h} v(x) \lambda_{x}$ and using $\left\|\lambda_{x}\right\|_{E} \sim h_{E}^{1 / 2}, h_{E} \leq h_{x}$ implies the result.

(ii). Suppose first that $E$ lies in $\bar{\Omega}_{\ell}$. Then $E \cap \bar{\Omega}_{\ell}=E$ and for both end points of $E$, we have:

$$
\left|\pi_{h} v(x)\right| \lesssim h_{x}^{-1}\left\|\tilde{v}_{\ell}\right\|_{\omega_{x}}+\left\|\nabla \tilde{v}_{\ell}\right\|_{\omega_{x}}
$$

The estimate is obtained as in (i). If $E$ is cut by the crack the discussion is the same.

(iii) and (iv). Straightforward (see (i) and (ii)).

Now we need to study the stability of the quasi-interpolation operator $\pi_{h}$ on the crack. We denote by $F_{h} \subset E_{h}$ the set of edges lying on the crack (these edges are the ones of the generalized elements on the crack).

Lemma 4.7. For all $v \in H^{1}(\Omega)$ and all edge $F \in F_{h}$ one has:

(i) If $F \subset T \in T_{h}$ where $T$ is totally enriched, then for $\ell=1$ and $\ell=2$, we have:

$$
\left\|\left(\pi_{h} v\right)_{\left.\right|_{\Omega_{\ell}}}\right\|_{F} \lesssim h_{F}^{1 / 2} h_{T}^{-1}\left\|\tilde{v}_{\ell}\right\|_{\omega_{T}}+h_{F}^{1 / 2}\left\|\nabla \tilde{v}_{\ell}\right\|_{\omega_{T}}
$$

(ii) If $F \subset T \in T_{h}$ where $T$ is partially enriched, then for $\ell=1$ and $\ell=2$, we have:

$$
\left\|\left(\pi_{h} v\right)_{\left.\right|_{\Omega_{\ell}}}\right\|_{F} \lesssim h_{F}^{1 / 2} h_{T}^{-1}\left\|\tilde{v}_{\ell}\right\|_{\omega_{T}}+h_{F}^{1 / 2}\left\|\nabla \tilde{v}_{\ell}\right\|_{\omega_{T}}+h_{F}^{1 / 2} h_{T}^{-1}\|v\|_{\omega_{T}}+h_{F}^{1 / 2}\|\nabla v\|_{\omega_{T}}
$$

(iii) If $F \subset T \in T_{h}$ where the crack tip lies in the interior of $T$, (then $\left(\pi_{h} v\right)_{\left.\right|_{\Omega_{1}}}=\left(\pi_{h} v\right)_{\left.\right|_{\Omega_{2}}}$ on $\left.F\right)$, we have:

$$
\left\|\pi_{h} v\right\|_{F} \lesssim h_{F}^{1 / 2} h_{T}^{-1}\|v\|_{\omega_{T}}+h_{F}^{1 / 2}\|\nabla v\|_{\omega_{T}}
$$


Proof: (i) and (ii). First we consider an edge $F=(a, b)$ which does not contain the crack tip and we fix $\ell=1$ or $\ell=2$. On $F$, we have

$$
\left(\pi_{h} v\right)_{\left.\right|_{\Omega_{\ell}}}=\left(\pi_{h} v\right)_{\left.\right|_{\Omega_{\ell}}}(a) \lambda_{a}+\left(\pi_{h} v\right)_{\left.\right|_{\Omega_{\ell}}}(b) \lambda_{b}
$$

where $\lambda_{a}$ and $\lambda_{b}$ are the edge basis functions at $a$ and $b$. Let $T=x_{1} x_{2} x_{3} \in T_{h}$ be the triangle containing $F$. Since $\left(\pi_{h} v\right)_{\left.\right|_{\Omega_{\ell}}}$ is constructed by the restriction of an affine extension on $T$, it is straightforward that $\left(\pi_{h} v\right)_{\Omega_{\ell}}(a)$ and $\left(\pi_{h} v\right)_{\left.\right|_{\Omega_{\ell}}}(b)$ are convex combinations of $\pi_{h} \tilde{v}_{\ell}\left(x_{i}\right)$. Depending on the fact that $x_{i}$ is enriched or not we have either:

or

$$
\left|\pi_{h} \tilde{v}_{\ell}\left(x_{i}\right)\right| \lesssim h_{x_{i}}^{-1}\left\|\tilde{v}_{\ell}\right\|_{\omega_{x_{i}}}+\left\|\nabla \tilde{v}_{\ell}\right\|_{\omega_{x_{i}}},
$$

$$
\left|\pi_{h} \tilde{v}_{\ell}\left(x_{i}\right)\right| \lesssim h_{x_{i}}^{-1}\|v\|_{\omega_{x_{i}}}+\|\nabla v\|_{\omega_{x_{i}}}
$$

Using $\left\|\lambda_{a}\right\|_{F} \sim\left\|\lambda_{b}\right\|_{F} \sim h_{F}^{1 / 2}$, implies the result in (i), (ii) and (iii).

\subsection{Error estimates}

We now consider the local error estimates in the $L^{2}$ norms.

Lemma 4.8. For all $v \in H^{1}(\Omega)$ and all $T \in T_{h}$ one has:

(i) if none of the nodes of $T$ is enriched then

$$
\left\|v-\pi_{h} v\right\|_{T} \lesssim h_{T}\|\nabla v\|_{\omega_{T}}
$$

(ii) if the three nodes of $T$ are enriched then for $\ell=1$ or $\ell=2$, we have:

$$
\left\|v-\pi_{h} v\right\|_{T \cap \Omega_{\ell}} \lesssim h_{T}\left\|\nabla \tilde{v}_{\ell}\right\|_{\omega_{T}}
$$

(iii) if one or two nodes of $T$ are enriched and if $\omega_{T}$ is cut by the crack (so $T \subset \bar{\Omega}_{\ell}$ for $\ell=1$ or $\left.\ell=2\right)$ we have:

$$
\left\|v-\pi_{h} v\right\|_{T} \lesssim h_{T}\left\|\nabla \tilde{v}_{\ell}\right\|_{\omega_{T}}
$$

(iv) if one or two nodes of $T$ are enriched and if $\omega_{T}$ contains the crack tip then for $\ell=1$ or $\ell=2$, we have:

$$
\left\|v-\pi_{h} v\right\|_{T \cap \Omega_{\ell}} \lesssim h_{T} \sqrt{-\ln \left(h_{T}\right)}\left(\left\|\nabla \tilde{v}_{\ell}\right\|_{\Omega}+\|\nabla v\|_{\Omega}\right)
$$

Proof: We first note that $\pi_{h}$ preserves the constant functions (note that we can suppose that constant functions are extended on the other side of the crack by the same constant functions). Hence, for any $v \in H^{1}(\Omega)$ and any constant function $c(x)=c$ we can write:

$$
v-\pi_{h} v=v-c-\pi_{h}(v-c) .
$$

(i) In this case

$$
\left\|v-\pi_{h} v\right\|_{T} \leq\|v-c\|_{\omega_{T}}+\left\|\pi_{h}(v-c)\right\|_{T} \lesssim h_{T}\|\nabla v\|_{\omega_{T}},
$$

where we use Lemma 4.3(i) and we choose $c=\left|\omega_{T}\right|^{-1} \int_{\omega_{T}} v(x) d x$ together with $h_{T} \sim h_{\omega_{T}}$.

(ii) We write, for any constant function $c$ :

$$
\begin{aligned}
\left\|v-\pi_{h} v\right\|_{T \cap \Omega_{\ell}} & \leq\left\|\tilde{v}_{\ell}-c\right\|_{\omega_{T}}+\left\|\pi_{h}(v-c)\right\|_{T \cap \Omega_{\ell}} \\
& \lesssim\left\|\tilde{v}_{\ell}-c\right\|_{\omega_{T}}+h_{T}\left\|\nabla \tilde{v}_{\ell}\right\|_{\omega_{T}} \\
& \lesssim h_{T}\left\|\nabla \tilde{v}_{\ell}\right\|_{\omega_{T}}
\end{aligned}
$$


where $c=\left|\omega_{T}\right|^{-1} \int_{\omega_{T}} \tilde{v}_{\ell}(x) d x$ and we conclude as in (i) using Lemma 4.3(ii).

(iii) As the previous cases.

(iv) This estimate is obtained as follows using Lemma 4.3(iv) and choosing $c=\left|\omega_{T}\right|^{-1} \int_{\omega_{T}} \tilde{v}_{\ell}(x) d x$ :

$$
\begin{aligned}
\left\|v-\pi_{h} v\right\|_{T \cap \Omega_{\ell}} & \leq\left\|\tilde{v}_{\ell}-c\right\|_{\omega_{T}}+\left\|\pi_{h}(v-c)\right\|_{T \cap \Omega_{\ell}} \\
& \lesssim\left\|\tilde{v}_{\ell}-c\right\|_{\omega_{T}}+\|v-c\|_{\omega_{T}}+h_{T}\left\|\nabla \tilde{v}_{\ell}\right\|_{\omega_{T}}+h_{T}\|\nabla v\|_{\omega_{T}} \\
& \lesssim\left\|\tilde{v}_{\ell}-v\right\|_{\omega_{T}}+h_{T}\left\|\nabla \tilde{v}_{\ell}\right\|_{\omega_{T}}+h_{T}\|\nabla v\|_{\omega_{T}} .
\end{aligned}
$$

Moreover, denoting by $1_{X}$ the characteristic function of the set $X$, we write

$$
\begin{aligned}
\left\|\tilde{v}_{\ell}-v\right\|_{\omega_{T}} & =\left\|\left(\tilde{v}_{\ell}-v\right) 1_{\omega_{T}}\right\|_{\Omega} \\
& \leq\left\|\tilde{v}_{\ell}-v\right\|_{L^{q}(\Omega)}\left\|1_{\omega_{T}}\right\|_{L^{\frac{2 q}{q-2}}(\Omega)} \\
& \lesssim h_{T}^{1-\frac{2}{q}}\left\|\tilde{v}_{\ell}-v\right\|_{L^{q}(\Omega)} \\
& \lesssim q^{\frac{1}{2}} h_{T}^{1-\frac{2}{q}}\left\|\tilde{v}_{\ell}-v\right\|_{1, \Omega} \\
& \lesssim q^{\frac{1}{2}} h_{T}^{1-\frac{2}{q}}\left\|\nabla\left(\tilde{v}_{\ell}-v\right)\right\|_{\Omega} \\
& \lesssim q^{\frac{1}{2}} h_{T}^{1-\frac{2}{q}}\left(\left\|\nabla \tilde{v}_{\ell}\right\|_{\Omega}+\|\nabla v\|_{\Omega}\right)
\end{aligned}
$$

where $2<q<\infty$ and we have used the Sobolev inequality $\|w\|_{L^{q}(\Omega)} \leq C q^{1 / 2}\|w\|_{1, \Omega}$, see e.g. [13]. We obtain the final estimate by choosing $q=-\ln \left(h_{T}\right)$.

Lemma 4.9. For all $v \in H^{1}(\Omega)$ and all edge $E$ of a triangle $T \in T_{h}$ one has:

(i) if both end points of $E$ are not enriched:

$$
\left\|v-\pi_{h} v\right\|_{E} \lesssim h_{E}^{1 / 2}\|\nabla v\|_{\omega_{E}},
$$

(ii) if both end points of $E$ are enriched then for $\ell=1$ or $\ell=2$, we have:

$$
\left\|v-\pi_{h} v\right\|_{E \cap \bar{\Omega}_{\ell}} \lesssim h_{E}^{1 / 2}\left\|\nabla \tilde{v}_{\ell}\right\|_{\omega_{E}}
$$

(iii) if only one end point of $E$ is enriched and if $\omega_{E}$ is cut by the crack (so $E \subset \bar{\Omega}_{\ell}$ for $\ell=1$ or $\ell=2$ ), we have:

$$
\left\|v-\pi_{h} v\right\|_{E} \lesssim h_{E}^{1 / 2}\left\|\nabla \tilde{v}_{\ell}\right\|_{\omega_{E}},
$$

(iv) if only one end point of $E$ is enriched and if $\omega_{E}$ contains the crack tip then for $\ell=1$ or $\ell=2$, we have:

$$
\left\|v-\pi_{h} v\right\|_{E \cap \bar{\Omega}_{\ell}} \lesssim h_{E}^{1 / 2} \sqrt{-\ln \left(h_{E}\right)}\left(\left\|\nabla \tilde{v}_{\ell}\right\|_{\Omega}+\|\nabla v\|_{\Omega}\right) .
$$

Proof: (i) Since $\pi_{h}$ preserves the constant functions we have for all $v \in H^{1}(\Omega)$ and all constant function $c(x)=c: v-\pi_{h} v=v-c-\pi_{h}(v-c)$. So by the scaled trace inequality (8) and Lemma 4.5(i), we obtain

$$
\left\|v-\pi_{h} v\right\|_{E} \leq\|v-c\|_{E}+\left\|\pi_{h}(v-c)\right\|_{E} \lesssim h_{E}^{-1 / 2}\|v-c\|_{\omega_{E}}+h_{E}^{1 / 2}\|\nabla v\|_{\omega_{E}},
$$

and we choose $c=\left|\omega_{E}\right|^{-1} \int_{\omega_{E}} v(x) d x$ together with $h_{E} \sim h_{\omega_{E}}$.

(ii, iii) As in the previous cases. 
(iv) This estimate is obtained as follows by using Lemma 4.5(iv) and choosing $c=\left|\omega_{E}\right|^{-1} \int_{\omega_{E}} \tilde{v}_{\ell}(x) d x$. We then achieve the same calculations as in Lemma 4.8(iv):

$$
\begin{aligned}
\left\|v-\pi_{h} v\right\|_{E \cap \bar{\Omega}_{\ell}} & \leq\left\|\tilde{v}_{\ell}-c\right\|_{E}+\left\|\pi_{h}(v-c)\right\|_{E \cap \bar{\Omega}_{\ell}} \\
& \lesssim h_{E}^{-1 / 2}\left\|\tilde{v}_{\ell}-c\right\|_{\omega_{E}}+h_{E}^{-1 / 2}\|v-c\|_{\omega_{E}}+h_{E}^{1 / 2}\left\|\nabla \tilde{v}_{\ell}\right\|_{\omega_{E}}+h_{E}^{1 / 2}\|\nabla v\|_{\omega_{E}} \\
& \lesssim h_{E}^{-1 / 2}\left\|\tilde{v}_{\ell}-v\right\|_{\omega_{E}}+h_{E}^{1 / 2}\left\|\nabla \tilde{v}_{\ell}\right\|_{\omega_{E}}+h_{E}^{1 / 2}\|\nabla v\|_{\omega_{E}} \\
& \lesssim h_{E}^{1 / 2} \sqrt{-\ln \left(h_{E}\right)}\left(\left\|\nabla \tilde{v}_{\ell}\right\|_{\Omega}+\|\nabla v\|_{\Omega}\right) .
\end{aligned}
$$

The next lemma consists of estimating the error committed by the averaging operator on the edges of the generalized elements located on the crack. The set of such edges is denoted $F_{h}$.

Lemma 4.10. For all $v \in H^{1}(\Omega)$ and all edge $F \in F_{h}$ one has:

(i) If $F \subset T \in T_{h}$ where $T$ is totally enriched, then for $\ell=1$ and $\ell=2$, we have:

$$
\left\|\left(v-\pi_{h} v\right)_{\Omega_{\ell}}\right\|_{F} \lesssim h_{T}^{1 / 2}\left\|\nabla \tilde{v}_{\ell}\right\|_{\omega_{T}}
$$

(ii) If $F \subset T \in T_{h}$ where $T$ is partially enriched, then for $\ell=1$ and $\ell=2$, we have:

$$
\left\|\left(v-\pi_{h} v\right)_{\Omega_{\Omega_{\ell}}}\right\|_{F} \lesssim h_{T}^{1 / 2} \sqrt{-\ln \left(h_{T}\right)}\left(\left\|\nabla \tilde{v}_{\ell}\right\|_{\Omega}+\|\nabla v\|_{\Omega}\right)
$$

(iii) If $F \subset T \in T_{h}$ where the crack tip lies in the interior of $T$, (then $\left(\pi_{h} v\right)_{\left.\right|_{\Omega_{1}}}=\left(\pi_{h} v\right)_{\left.\right|_{\Omega_{2}}}$ on $\left.F\right)$, we have:

$$
\left\|v_{\left.\right|_{\Omega_{\ell}}}-\pi_{h} v\right\|_{F} \lesssim h_{T}^{1 / 2}\|\nabla v\|_{\omega_{T}}
$$

Proof: (i) Since $\pi_{h}$ preserves the constant functions we have for all $v \in H^{1}(\Omega)$ and all constant function $c(x)=c: v-\pi_{h} v=v-c-\pi_{h}(v-c)$. By the generalized scaled trace inequality (see $\left.[15,17]\right)$ :

$$
\|w\|_{F} \lesssim h_{T}^{-1 / 2}\|w\|_{T}+h_{T}^{1 / 2}\|\nabla w\|_{T}
$$

and Lemma 4.7, we obtain

$$
\begin{aligned}
\left\|\left(v-\pi_{h} v\right)_{\left.\right|_{\Omega_{\ell}}}\right\|_{F} & \leq\left\|\tilde{v}_{\ell}-c\right\|_{F}+\left\|\pi_{h}\left(\tilde{v}_{\ell}-c\right)\right\|_{F} \\
& \lesssim h_{T}^{-1 / 2}\left\|\tilde{v}_{\ell}-c\right\|_{T}+h_{T}^{1 / 2}\left\|\nabla \tilde{v}_{\ell}\right\|_{T}+h_{F}^{1 / 2} h_{T}^{-1}\left\|\tilde{v}_{\ell}-c\right\|_{\omega_{T}}+h_{F}^{1 / 2}\left\|\nabla \tilde{v}_{\ell}\right\|_{\omega_{T}} .
\end{aligned}
$$

Hence the conclusion. The proof of (ii) and (iii) are the same as previously.

\section{ERror ESTIMATORS}

\subsection{Definition of the residual error estimators}

Writing $u_{h}=u_{h, r}+\chi u_{h, s}$ as in (3), the exact element residual is defined by

$$
R_{G}=f+\Delta u_{h}=f+\Delta\left(\chi u_{h, s}\right)=f+c \Delta\left(\chi r^{1 / 2} \sin (\theta / 2)\right)
$$

on each generalized element $G \in G_{h}$. Since $\Delta\left(r^{1 / 2} \sin (\theta / 2)\right)=0$ we deduce that the expression $\Delta\left(\chi r^{1 / 2} \sin (\theta / 2)\right)$ vanishes excepted on the elements (distant from the crack tip) having an nonempty intersection with the ring shaped area where $\chi$ maps onto $(0,1)$. 
Definition 5.1 (Residual error estimator). Let $G \in G_{h}$ and $T \in T_{h}$ be the triangle containing $G$. The local and global residual error estimators are defined by

$$
\begin{aligned}
\eta_{1 G} & =h_{T} C\left(h_{T}\right)\left\|f+\Delta\left(\chi u_{h, s}\right)\right\|_{G}, \\
\eta_{2 G} & \left.=h_{T}^{1 / 2} D\left(h_{T}\right)\left(\sum_{E \in E_{G}^{\text {int }} \cup E_{G}^{N} \cup E_{G}^{C}} \|\left[\partial u_{h} / \partial n\right]\right]_{E} \|_{E}^{2}\right)^{1 / 2}, \\
\eta_{G} & =\left(\eta_{1 G}^{2}+\eta_{2 G}^{2}\right)^{1 / 2}, \\
\eta & =\left(\sum_{G \in G_{h}} \eta_{G}^{2}\right)^{1 / 2},
\end{aligned}
$$

where $C\left(h_{T}\right)=\sqrt{-\ln \left(h_{T}\right)}$ for the elements in case (iv) of Lemma 4.8, otherwise $C\left(h_{T}\right)=1$ and $D\left(h_{T}\right)=$ $\sqrt{-\ln \left(h_{T}\right)}$ for the elements in case (iv) of Lemma 4.9 or in case (ii) of Lemma 4.10, otherwise $D\left(h_{T}\right)=1$.

Remark 5.2. The presence of the $\ln \left(h_{T}\right)$-terms in the estimator results from technical reasons and appears only for a bounded number (independent of the mesh) of elements near the crack tip. From a numerical point of view, these $\ln \left(h_{T}\right)$-terms are negligible. In the case of a standard finite element method with coinciding finite element meshes on the crack we have $G_{h}=T_{h}$ and $G=T$ (obviously the XFEM is not a generalization of a standard finite element method with noncoinciding finite element meshes on the crack). In the case of coinciding meshes on the crack it is easy to show that the cases involving the $\ln \left(h_{T}\right)$-terms disappear (i.e., $C\left(h_{T}\right)=D\left(h_{T}\right)=1$ ) and we recover the classical residual estimator (see e.g., [4, 25]).

\subsection{Upper error bound}

Theorem 5.3. Let $u \in V$ be the solution of (2) and let $u_{h} \in V_{h}$ be the solution of (4). Then

$$
\left\|\nabla\left(u-u_{h}\right)\right\|_{\Omega} \lesssim \eta .
$$

Proof: Denoting the error by

$$
e=u-u_{h},
$$

we have, according to (2) and (4):

$$
\begin{aligned}
\|\nabla e\|_{\Omega}^{2} & =\int_{\Omega} \nabla u \cdot \nabla\left(u-u_{h}\right)-\int_{\Omega} \nabla u_{h} \cdot \nabla\left(u-u_{h}\right) \\
& =\int_{\Omega} f\left(u-u_{h}\right)-\int_{\Omega} \nabla u_{h} \cdot \nabla\left(u-u_{h}\right) \\
& =\int_{\Omega} f\left(u-v_{h}\right)-\int_{\Omega} \nabla u_{h} \cdot \nabla\left(u-v_{h}\right), \quad \forall v_{h} \in V_{h} .
\end{aligned}
$$


Splitting up the integrals on each generalized element $G \in G_{h}$ and writing $u_{h}=u_{h, r}+\chi u_{h, s}$, we arrive at

$$
\begin{aligned}
\|\nabla e\|_{\Omega}^{2}= & \sum_{G \in G_{h}} \int_{G} f\left(u-v_{h}\right) \\
& -\sum_{G \in G_{h}} \int_{G} \nabla u_{h, r} \cdot \nabla\left(u-v_{h}\right)-\sum_{G \in G_{h}} \int_{G} \nabla\left(\chi u_{h, s}\right) \cdot \nabla\left(u-v_{h}\right) \\
= & \sum_{G \in G_{h}} \int_{G}\left(f+\Delta\left(\chi u_{h, s}\right)\right)\left(u-v_{h}\right) \\
- & \left.\sum_{E \in E_{h}^{\text {int }}} \int_{E} \llbracket \partial u_{h, r} / \partial n\right]_{E}\left(u-v_{h}\right)-\sum_{E \in E_{h}^{N} \cup E_{h}^{C}} \int_{E} \frac{\partial u_{h}}{\partial n_{E}}\left(u-v_{h}\right), \quad \forall v_{h} \in V_{h},
\end{aligned}
$$

where we have used the Green formula on each generalized element (note that although the triangle containing the crack tip has a boundary which is not Lipschitz, it can be divided in two parts by using a straight extension of the crack and then one can use separately Green's formula on each part to obtain the desired result) as well as $\Delta u_{h, r}=0$ on $G$ and $\left[\left[\partial\left(\chi u_{h, s}\right) / \partial n\right]_{E}=0\right.$ for all $E \in E_{h}^{i n t}$.

At this stage we fix the choice of $v_{h}$. We set

$$
v_{h}=u_{h}+\pi_{h}\left(u-u_{h}\right) .
$$

We consider (11): with the above choice we are able to estimate each term of the right-hand side of the previous expression. Cauchy-Schwarz inequality implies

$$
\sum_{G \in G_{h}} \int_{G}\left(f+\Delta\left(\chi u_{h, s}\right)\right)\left(u-v_{h}\right) \leq \sum_{G \in G_{h}}\left\|f+\Delta\left(\chi u_{h, s}\right)\right\|_{G}\left\|u-v_{h}\right\|_{G} .
$$

Therefore it remains to estimate $\left\|u-v_{h}\right\|_{G}$ for any generalized element $G$. Let $T \in T_{h}$ be the triangle containing $G$. Using Lemma 4.8, we obtain for the triangles considered in cases (i)-(iii):

$$
\left\|u-v_{h}\right\|_{G}=\left\|e-\pi_{h} e\right\|_{G} \lesssim h_{T}\|\nabla e\|_{\omega_{T}}
$$

or

$$
\left\|u-v_{h}\right\|_{G}=\left\|e-\pi_{h} e\right\|_{G} \lesssim h_{T}\|\nabla \tilde{e}\|_{\omega_{T}},
$$

where $\tilde{e}$ is an extension of the error across the crack (see (5), (6)). If $T$ belongs to the finite set of triangles (iv) in Lemma 4.8, we have

$$
\left\|u-v_{h}\right\|_{G}=\left\|e-\pi_{h} e\right\|_{G} \lesssim h_{T} \sqrt{-\ln \left(h_{T}\right)}\left(\|\nabla \tilde{e}\|_{\Omega}+\|\nabla e\|_{\Omega}\right) .
$$

So, depending on the cases (i)-(iv) of Lemma 4.8 and using estimates (12)-(14), we can write

$$
\begin{aligned}
& \sum_{G \in G_{h}} \int_{G}\left(f+\Delta\left(\chi u_{h, s}\right)\right)\left(u-v_{h}\right) \\
\lesssim & \left(\sum_{G \in G_{h}, \text { cases }(i)-(i i i)} h_{T}^{2}\left\|f+\Delta\left(\chi u_{h, s}\right)\right\|_{G}^{2}\right)^{1 / 2}\left(\sum_{G \in G_{h}, \text { cases }(i)-(i i i)}\left(\|\nabla \tilde{e}\|_{\omega_{T}}+\|\nabla e\|_{\omega_{T}}\right)^{2}\right)^{1 / 2} \\
& +\left(\sum_{G \in G_{h}, \text { case }(i v)} h_{T}^{2}\left(-\ln \left(h_{T}\right)\right)\left\|f+\Delta\left(\chi u_{h, s}\right)\right\|_{G}^{2}\right)^{1 / 2}\left(\sum_{G \in G_{h}, \text { case }(i v)}\left(\|\nabla \tilde{e}\|_{\Omega}+\|\nabla e\|_{\Omega}\right)^{2}\right)^{1 / 2} \\
\lesssim & \eta\|\nabla e\|_{\Omega} .
\end{aligned}
$$


Let us now pass to the estimate of the remaining terms: as before the application of Cauchy-Schwarz inequality leads to

$$
\begin{aligned}
& -\sum_{E \in E_{h}^{i n t}} \int_{E}\left[\left[\partial u_{h, r} / \partial n\right]\right]_{E}\left(u-v_{h}\right)-\sum_{E \in E_{h}^{N} \cup E_{h}^{C}} \int_{E} \frac{\partial u_{h}}{\partial n_{E}}\left(u-v_{h}\right) \\
= & -\sum_{E \in E_{h}^{N} \cup E_{h}^{i n t} \cup E_{h}^{C}} \int_{E}\left[\left[\partial u_{h} / \partial n\right]_{E}\left(e-\pi_{h} e\right)\right. \\
\leq & \left.\sum_{E \in E_{h}^{N} \cup E_{h}^{i n t} \cup E_{h}^{C}} \|\left[\partial u_{h} / \partial n\right]\right]_{E}\left\|_{E}\right\| e-\pi_{h} e \|_{E} .
\end{aligned}
$$

Using the Lemmas 4.9(i,ii,iii) and 4.10(i,iii) and denoting by $T \in T_{h}$ a triangle containing $E$, we obtain

$$
\left\|e-\pi_{h} e\right\|_{E} \lesssim h_{T}^{1 / 2}\left(\|\nabla \tilde{e}\|_{\omega_{T}}+\|\nabla e\|_{\omega_{T}}\right) .
$$

If $E$ belongs to the finite set of triangles in Lemma 4.9(iv) or in Lemma 4.10(ii), we have

$$
\left\|e-\pi_{h} e\right\|_{E} \lesssim h_{T}^{1 / 2} \sqrt{-\ln \left(h_{T}\right)}\left(\|\nabla \tilde{e}\|_{\Omega}+\|\nabla e\|_{\Omega}\right) .
$$

Using estimates (17) and (18) with (16) as well as (15) ends the proof of the theorem.

\section{Concluding Remarks And Perspectives}

In this work we perform a residual a posteriori error analysis of the extended finite element method applied to Poisson's problem. To our knowledge, this is the first residual a posteriori analysis for XFEM. We build an appropriate quasi-interpolation operator which allows us to obtain an upper bound of the discretization error. The proof of a lower bound remains an open problem and the forthcoming numerical experiments in [19] using Getfem (see [14]) will give more information on the performances of this estimator.

\section{REFERENCES}

[1] R.A. Adams, Sobolev spaces, Academic Press, 1975.

[2] I. Babuška and W. Rheinboldt, Error estimates for adaptive finite element computations, SIAM J. Numer. Anal., 15 (1978) 736-754.

[3] C. Bernardi and V. Girault, A local regularisation operator for triangular and quadrilateral finite elements, SIAM J. Numer. Anal., 35 (1998) 1893-1916.

[4] C. Bernardi, B. Métivet and R. Verfürth, Analyse numérique d'indicateurs d'erreur, Report 93025, Laboratoire d'analyse numérique, Université Paris VI, 1993.

[5] S.C. Brenner and L.R. Scott, The mathematical theory of finite element methods, Springer-Verlag, 2002.

[6] E. Chahine, P. Laborde and Y. Renard, A quasi-optimal convergence result for fracture mechanics with XFEM, C. R. Acad. Sci. Paris, 342 (2006) 527-532.

[7] E. Chahine, P. Laborde and Y. Renard, Crack tip enrichment in XFEM using a cut-off function, to appear in Int. J. Numer. Meth. Engng.

[8] Z. Chen and R.H. Nochetto, Residual type a posteriori error estimates for elliptic obstacle problems, Numer. Math., 84 (2000) $527-548$.

[9] P.G. Ciarlet, The finite element method for elliptic problems, in Handbook of Numerical Analysis, Volume II, Part 1, Eds. P.G. Ciarlet and J.-L. Lions, North Holland, 17-352, 1991.

[10] P. Clément, Approximation by finite element functions using local regularization, RAIRO Anal. Numer., 2 (1975) 77-84.

[11] A. Ern and J.-L. Guermond, Eléments finis : théorie, applications, mise en oeuvre, Springer-Verlag, 2001.

[12] L. Evans, Partial differential equations, American Mathematical Society, Providence, Rhode island, 1999.

[13] H. Fujita and T. Susuki, Evolution problems, in Handbook of Numerical Analysis, Volume II, Part 1, Eds. P.G. Ciarlet and J.-L. Lions, North Holland, 791-927, 1991. 
[14] GETFEM++, www-gmm.insa-toulouse.fr/getfem/.

[15] P. Grisvard, Elliptic problems in nonsmooth domains, Pitman, Boston-London-Melbourne, 1985.

[16] P. Grisvard, Problèmes aux limites dans les polygones - mode d'emploi. EDF Bull. Direction Etudes Rech. Sér. C. Math. Inform. 1. MR 87g:35073, 21-59, 1986.

[17] J. Haslinger and Y. Renard, A new fictitious domain approach inspired by the extended finite element method, submitted.

[18] S. Hilbert, A mollifier useful for approximations in Sobolev spaces and some applications to approximating solutions of differential equations, Math. Comp., 27 (1973) 81-89.

[19] V. Lleras, Thesis, in preparation.

[20] N. Moës and T. Belytschko, XFEM: nouvelles frontières pour les éléments finis, Revue européenne des éléments finis (Calcul des structures GIENS'01), 11 (2002) 305-318.

[21] N. Moës, J. Dolbow and T. Belytschko, A finite element method for cracked growth without remeshing, Int. J. Numer. Meth. Engng., 46 (1999) 131-150.

[22] L.R. Scott and S. Zhang, Finite element interpolation of nonsmooth functions satisfying boundary conditions, Math. Comp., 54 (1990) 483-493.

[23] G. Strang, Approximation in the finite element method, Numer. Math., 19 (1972) 81-98.

[24] G. Strang, G. Fix, An analysis of the finite element method, Prentice-Hall, Englewood Cliffs, 1973.

[25] R. Verfürth, A review of a posteriori error estimation and adaptative meshrefinement techniques, Wiley and Teubner, Chichester and Stuttgart, 1996. 